\title{
A Review of Using Support Vector Machine Theory to Do Stock Forecasting
}

\author{
Meizhen Liu ${ }^{1, \text { a) }}$, Chunmei Duan ${ }^{2, \text { b) }}$ \\ ${ }^{1}$ School of Shandong Normal University, Jinan 250000, China. \\ ${ }^{2}$ School of Shandong Normal University, Jinan 250000, China. \\ a) 18353361502@163.com, ${ }^{\text {b) }}$ cmduan@sdnu.edu.cn
}

\begin{abstract}
SVM) is developed based on statistical learning theory new method, its training algorithm is essentially a problem of solving the quadratic programming. This paper summarizes the basic principle of SVM, and then use SVM to stock prediction research status at home and abroad were reviewed, analyzed using SVM analysis, stock price, stock index also simple analysis of financial condition, finally, the existing problems and development trend in this field were discussed.
\end{abstract}

Key words: statistical learning theory; Support vector machine (SVM); Stock prediction.

\section{STOCK PRICE INDEX}

Support vector machine (SVM) is a new general machine learning method developed in recent years. It can better solve the small sample, nonlinear Dimensional disasters and local minima [1-2] and other problems have been widely used in many fields such as pattern classification [3-4] and regression problems [5-6].

M Kumar and M Thenmozhi (2006) [7] illustrate that random forest and support vector machine (SVM) are specific machine learning methods and predict the stock market and price of financial instruments. The stock index prediction based on the mixed model is an important breakthrough in stock prediction. Ju-Jie Wang (2011) [8] uses two models to forecast the stock index, and proposes a hybrid method of ARIMA and SVM, and a genetic algorithm (GA). In this regard, M. Karazmodeh (2013) [9] analyzes the relationship between PSO and IPSO. The main difference is that the area of research using technical analysis of different indicators is used as an input function, the prediction of this model is more accurate and more valuable. An important improvement. LJ Kao (2013) [10] also elaborated a hybrid method for stock index forecasting, combined MARS and SVR based on wavelet feature extraction, multivariable adaptive regression spline (MARS) and support.

Vector regression (SVR) is an integrated stock price prediction model. This model not only solves the wavelet subsequence selection problem, but also improves the prediction accuracy. Xie Yimeng (2017) [11] described the time series model and the risk value, established a conditional heteroskedasticity model, and used the VaR value based on the CARCH model to describe the actual situation of the stock market risk, Huang Qing (2017) [12] combined the support vector machine method in machine learning with the Fama-Fench three-factor model to construct a new quantitative investment strategy and conducted an empirical analysis using A shares.

\section{STOCK PRICE}

Yukun Bao et al. (2006) [13] used support vector regression to predict stock prices and formed the basis for financial investment decisions. The feasibility of predicting stock prices. Liu Xiaolan (2012) [14] proposed a stock price forecasting model based on an improved support vector machine and optimized the parameters of the support vector machine. Lai Shaoyong (2013) [15] used wavelet support vector machine (SVM) method to forecast financial 
market data and used a combination of wavelet support vector machine and multivariate forecasting techniques to apply it to the prediction of stock prices. DH Nguyen, MT Le et al. (2014) [16] proposed a model to predict stock prices by combining self-organizing map (SOM) and fuzzy support vector machine (f-SVM).

Based on the original data extracted from fuzzy rules, combined with statistical machines Learning model method for stock price forecasting. Lin Qi et al. (2015) [17] proposed a method based on least squares support vector machine (SVM) and stock price data for phase space reconstruction based on the method of stock price reconstruction based on least squares method. Huang Tong-Yuan and Chen Fang-Fang (2016) [18] selected the optimal radial basis kernel function and then used the grid-seeking genetic algorithm and particle swarm algorithm to compare the best kernel function parameters.

\section{FINANCIAL STATEMENTS, CREDITS AND PORTFOLIOS}

In the field of investment research and application, hybrid securities also choose to use genetic algorithms and support vector regression models. En Yao, Yu Anle (2012) [19] introduced the idea of fuzzy membership on the basis of approximate support vector machine, it is also possible to reduce the interference of singular points and noise on the model, thereby further improving the classification discrimination ability. Pan Yangdi (2013) [20] based on the support vector machines for China's listed companies to carry out delisting risk early warning research, and China's motherboard and small and medium board manufacturing industry listed companies as examples for empirical research.

Pan Wenwen (2014) [21] established an SVM integration model for bank crisis assessment by applying SVM integration theory, classified bank crisis early warning evaluation indicators according to evaluation grades and used stochastic simulation to generate samples for future development of banking system operations. Luo Xiaoguang and Kong Hui (2015) [22] applied the stepwise discriminant analysis method to the selection of model variables to improve the efficiency of the model. At the same time, in order to avoid arbitrary selection of parameters, a particle swarm optimization algorithm was used to optimize the parameters of the support vector machine. Yang Qingxi (2016)[23] adjusted the fuzzy measures when the external environment may change greatly, taking into account the credibility of the output of each single classifier, the degree of similarity between the output of the classifiers, and the classifier itself. With different characteristics, in the financial crisis pre-answer model, earnings management is considered and a new fuzzy measure learning method is introduced to improve performance. Liu Shuang (2017) [24] chooses support vector machine as a reference model for calculation and comparison, which better approximates the daily closing price of Xinfu A-plus A stock index futures. Precise forecasting helps to increase the winning ratio and profitability of trading strategies. Space, while reducing the risk of price fluctuations.

\section{SUMMARY}

Statistical learning theory systematically studies machine learning problems, especially statistical learning in the case of finite samples. The SVM generated under this theoretical framework is a new general machine learning method, the theory and application of SVM algorithm have made great progress. the field needs further development and improvement. as to improve the prediction accuracy of the model and to have more effect on the stock prediction, so that it can be better Guide the operation of the stock market and gain profits.

\section{ACKNOWLEDGMENTS}

This research was supported by the National Nature Science Foundation of China (61502284,61602282).

\section{REFERENCES}

1. VAPNIK V. The nature of statistical learning theory[M].New York: Springer Verlag, 2000.

2. CRISTIANININ, SHAWE T J. An introduction to support vector machine [M]. New York: Cambridge University Press, 2000.

3. DOUMPOS M, ZOPOUNIDIS C. Additive support vector Machines for pattern classification[J]. IEEET ranson Systems, Man, and Cybernetics: Part B, 2007, 37(3): 540-550.

4. JAYADEVA, KHEMCHANDANIR, CHANDRA S. Twin support vector machines for pattern classification[J]. IEEE Transon Pattern Analysis and Machine Intelligence, 2007,29(5): 905-910. 
5. Wu Zhili, LIChunhung, JOSEPH K, etal. Locatione stimation via support vector regression[J]. IEEE Transon MobileComputing, 2007, 6(3): 311-321.

6. HAO Peiyi, CHIANG JH. Fuzzyregression analysisby support vector learning approach[J]. IEEETransonFuzzy Systems, 2008, 16(2): 428- 441.

7. M Kumar ,M Thenmozhi.The analysis of decomposition methods for support vector machines[J]. IEEE Transon Neural Networks, 2006, 11(4): 1003- 1008.

8. Ju-Jie Wanga. Stock index forecasting based on a hybrid model Stock index forecasting based on a hybrid model[J]. Omega,2011.

9. M. Karazmodeh, S. Nasiri, and S. Majid Hashemi, Stock Price Forecasting using Support Vector Machines and Improved Particle Swarm Optimization[J]. 2013.

10. ]LK Kao, Stock Price Combination Forecast Model Based on Regression Analysis and SVM,(Applied Mechanics \& Materials) ,2010.39:14-18.

11. Yimeng Xie. Analysis of Stock Market by Support Vector Machine and GARCH Family Model[D].2016.

12. Qing Huang. Study on Application of Support Vector Machines in China's A-share Market Quantitative Strategy_B Based on Fama-Fench Three-Factor Model[J], Time Finance.2017.

13. Yukun Bao. A Hybrid ARIMA and Support Vector Machines Model in Stock Price Forecasting [J]. Omega,2006, (33): 497 - 505.

14. Xiaolan Liu. Study on Stock Price Forecasting Based on Improved Support Vector Machine[J]. Agriculture Network Information .2012.

15. Shaoyong Lai, Ting Liu. Wavelet Support Vector Machine Theory and Its Application in Stock Index Prediction[D].2013.

16. ]MT Le,DH Nguyen .A two-stage architecture for stock price forecasting by combining SOM and fuzzySVM,2014.

17. ] Lin Qi. Stock price forecast based on LS-SVM phase space reconstruction [J]. IEEETrans on Circuits and Systems, 215, 51(5): 234-240.

18. Tongyi Huang, Fangfang Chen. Application of Kernel Function Based on SVM Stock Price Prediction[J].2016

19. XiaoYao, Lean Yu. Fuzzy Approximation Support Vector Machine Model and Its Application in Credit Risk Assessment[J]. System Engineering Theory \& Practice.2012.

20. Yangdi Pan. A Study on Early Warning Methods of Listed Companies' Delisting Risk-A Case Study of Listed Companies in China's Manufacturing Industry [D].2013.

21. Wenwen Pan. SVM Integrated Warning for Non-linear System of Bank Crisis in China[J]. Financial Reform.2014.

22. Xiaoguang Luo, Hui Kong. Discrimination Model of Enterprise's Debt Loss Rate Based on PSO_SVM[J]. Investment Research. 2015.

23. Qingyu Yang. Research on Dynamic Fuzzy Integral Support Vector Machine Integration Method and Application for Financial Crisis Early Warning[D].2016.

24. Shuang Liu. Application of Extreme Learning Machine Xinhua FTSE A50 Stock Index Futures Trading[D].2017. 